\title{
Report on Design, Development, and Characterization of a Coaxial Resonator Based Single-gap Gridless Multiharmonic Buncher
}

\author{
E. Pozdeyev, J. Brandon, N. Bultman, X. Rao, R. York, Q. Zhao
}

$2 / 11 / 2013$

\section{Scope}

This technical report describes the design of a gridless multiharmonic buncher funded by the DOE grant DE-FG02-08ER41553. The report also presents results of RF modeling and testing.

\section{Requirements}

The design of the multiharmonic buncher is determined by FRIB requirements. The buncher will bunch stable ion beams for injection into the FRIB RFQ to minimize the longitudinal beam emittance growth. The design beam energy is fixed at $12 \mathrm{keV} / \mathrm{u}$ while the beam charge state $\mathrm{Q} / \mathrm{A}$ can vary from $1 / 3$ to $1 / 7$. The buncher operates at a fundamental frequency of $40.25 \mathrm{MHz}$. Two higher harmonics, $80.5 \mathrm{MHz}$ and $120.75 \mathrm{MHz}$ are used to linearize the voltage ramp. The typical accelerating voltage for a uranium beam, including the time-of-flight factor, is $1271 \mathrm{~V}, 456 \mathrm{~V}$, and $150 \mathrm{~V}$ for $40.25 \mathrm{MHz}, 80.5 \mathrm{MHz}$, and $120.75 \mathrm{MHz}$ harmonics respectively. The voltage will be scaled with the charge state of an accelerated beam. The accelerated electrical beam current is expected to be approximately equal to $0.5 \mathrm{~mA}$ for all ion beams between oxygen and uranium.

\section{Mechanical Design}

The mechanical design of the multiharmonic buncher described in this report is based on the design of the ReA3 buncher [1] but includes modifications and improvements. Similar to the ReA3 buncher, the FRIB MHB uses two quarterwave resonators. Instead of drift tube electrodes terminated with a mesh grid, the FRIB MHB uses conical gridless electrodes that effectively form a single accelerating gap. Several other updates and improvements were implemented based on the experience with the ReA3 multiharmonic buncher. A detailed description of the buncher is provided below.

\section{Final drawings}

The final drawings of the buncher were posted on the FRIB document server (DCC). The final drawings package includes 58 drawings, 12 assemblies, and 54 individual parts. Some parts don't have individual drawings if they are welded inseparable. In this case, they are fully dimensioned in the assembly drawings. The drawings in DCC are numbered from T30505-MDE-0001-0000 through T30505-MDE-00010094, with the top level assembly drawing T30505-MDE-0001-0000. There are additional 17 drawings under T30505-MDE-0005, used for welding, assembly and test fixtures.

\section{General description of the mechanical assembly}

The buncher assembly utilizes two quarter wave resonators with a single gridless gap as a show in Figure 1. One resonator (the longer one) contains $40.25 \mathrm{MHz}$ and $120.75 \mathrm{MHz}$ harmonics and the other (shorter one) contains the $80.5 \mathrm{MHz}$ harmonic. Concentric conical accelerating electrodes at the end of the quarter wave resonators form a single accelerating gap. The resonators include power coupler, 
tuners, and a field probes, one of each per RF mode. The flange, which the resonators are attached to, have four precise pinholes for alignment targets. The relative position of the electrodes will be defined relatively to the alignment pinholes prior to installation for alignment purposes.

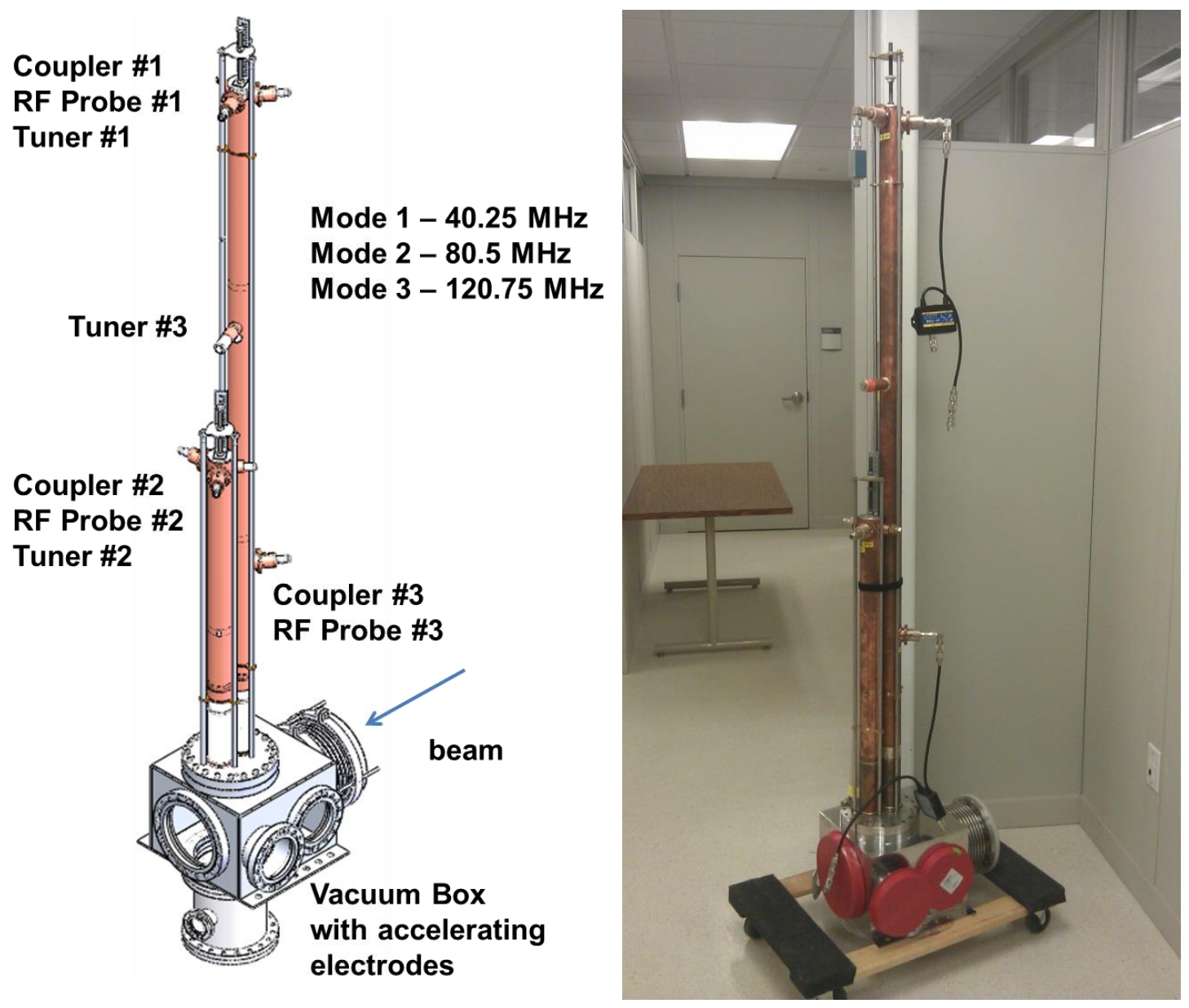

Figure 1: FRIB multi-harmonic buncher: design model and photograph of the full assembly. The buncher consists of two quarter wave resonators, a longer one with 40.25 and $120.75 \mathrm{MHz}$ modes, and a shorter one with an $80.5 \mathrm{MHz}$ mode. The accelerating electrodes are situated in the vacuum box.

\section{Resonators}

The two quarter wave resonators are similar to each other in design and differ only in length and location of couplers, tuners, and probes. The outer wall of the quarter wave resonators was made of 2" OD copper tubing. The central electrode of the resonators was made of 1" OD tubing. Each resonator is made of two sections, fixed and movable, to allow for length and frequency adjustability. The sections overlap with a contact provided by RF silver-plated fingers. The adjustability range is \pm 1 ". Figure 2 shows a cross section of the resonators along with the zoom-in view of the overlap area. 


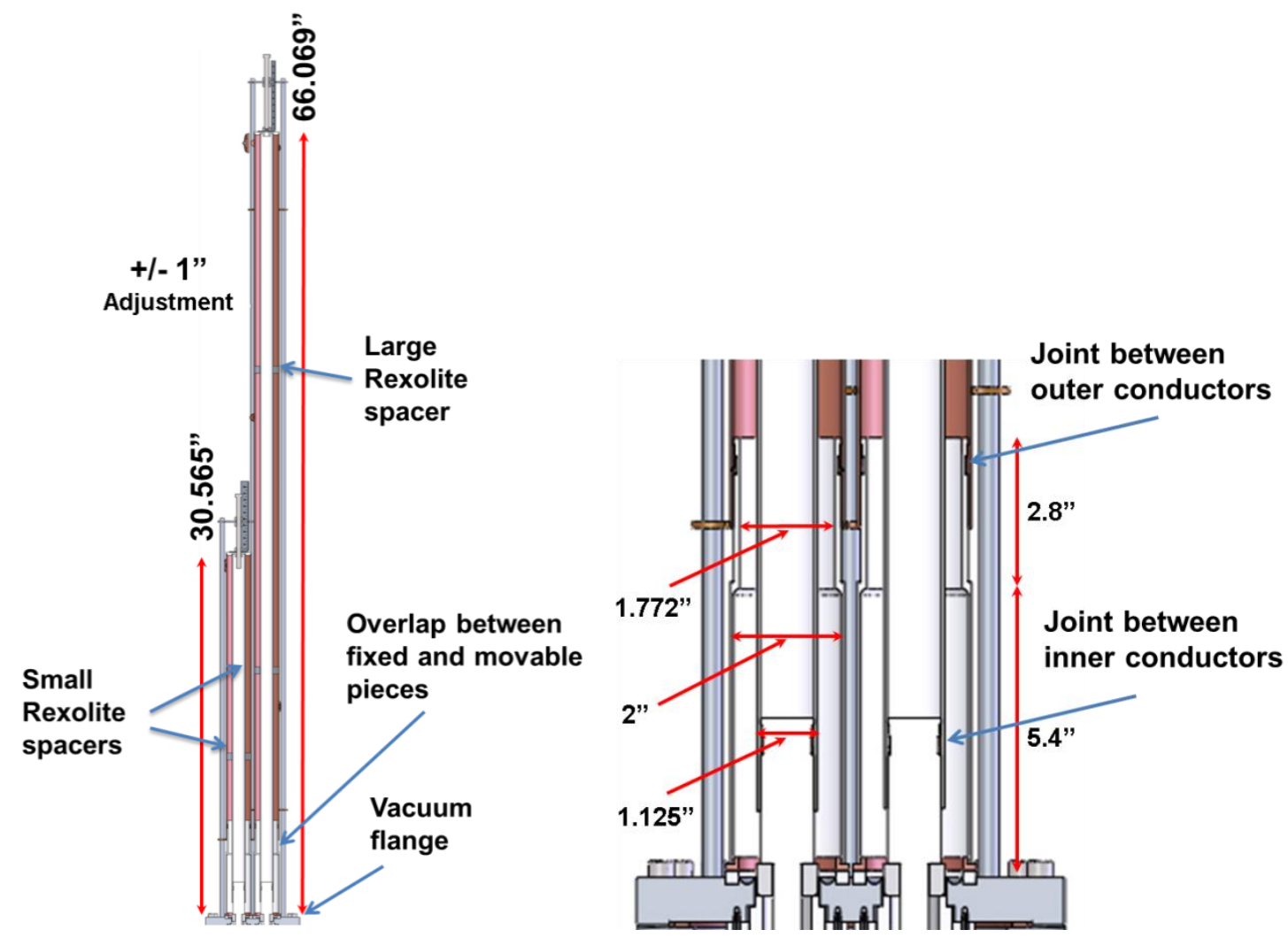

Figure 2: MHB quarter wave resonators. Left figure shows cross sectional view of the resonators. The figure on the right shows a zoom-in view of the overlap area between movable and fixed parts of the resonators.

Rexolite spacers are inserted into the QWRs to center the inner electrodes: two in the long resonator and one in the short resonator. The spacers have slots for a key tool used to securely control the position of the spacers. The upper spacer in the long resonator had additional material to adjust the frequency of the $120.75 \mathrm{MHz}$. Figure 3 shows the Rexolite spaces. Azimuthal slots in the spacers are provided for the key tool used to manipulate the spacers inside the resonators.

Small Rexolite Spacer

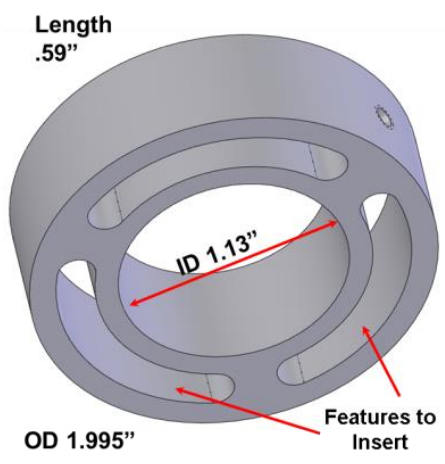

Large Rexolite Spacer

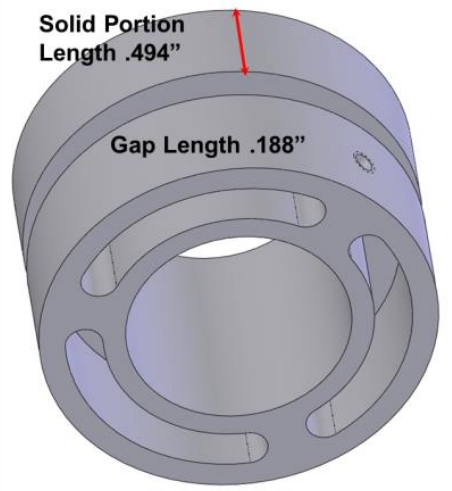

Figure 3: Rexolite spacer to center inner electrodes: small (left) and large (right). The length of the solid part of the larger spacer was machined to adjust the frequency of the $\mathbf{1 2 0 . 7 5}$ mode. 
Commercially available ceramic breaks provided vacuum seal for QWR central electrodes. The breaks were welded to bi-metal flanges that were brazed, in turn, to the central electrodes as shown in Figure 4. Small Rexolite washers/spacers were used to position central electrodes inside the ceramic breaks.

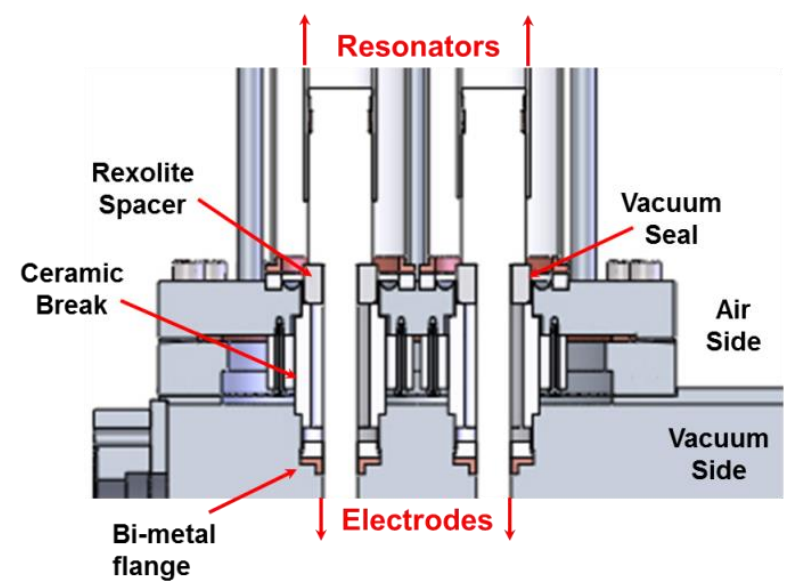

Figure 4: Zoom-in picture of the vacuum seal.

\section{Conical accelerating electrodes}

The main distinction of the FRIB MHB design from the ReA3 design is the shape of the accelerating electrodes. The FRIB buncher electrodes, shown in Figure 5, are conical shapes machined from solid copper. The distance between the electrodes is $4 \mathrm{~mm}$. The diameter of the smaller opening is $10 \mathrm{~mm}$ and the diameter of the larger opening is $1.9^{\prime \prime}(48.26 \mathrm{~mm})$. The pair of the electrodes effectively forms a single gap between the electrodes. The effect of the accelerating electric fields at the entrance and exit openings is zeroed out because of a large extent of the field area and slow beam velocity. The electrodes have small inter-electrode capacitance, providing negligible coupling between the quarter wave resonators. The electrodes are exchangeable in case different dimensions of the electrode opening are needed. Alignment of the electrodes is achieved using dowel pin holes at the top of the electrodes.

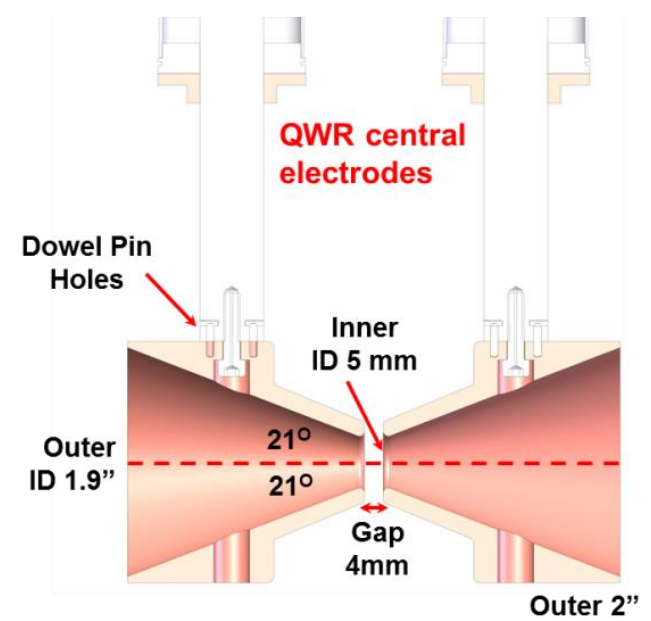

Figure 5: Accelerating electrodes 


\section{Power coupler design}

MHB uses three power couplers, one per mode, to provide RF power. Power couplers are copper assemblies with Type- $\mathrm{N}$ connectors. The outer bodies of the couplers were brazed to the resonators. The coupler of the $40.25 \mathrm{MHz}$ mode had a double loop to increase coupling to the mode.
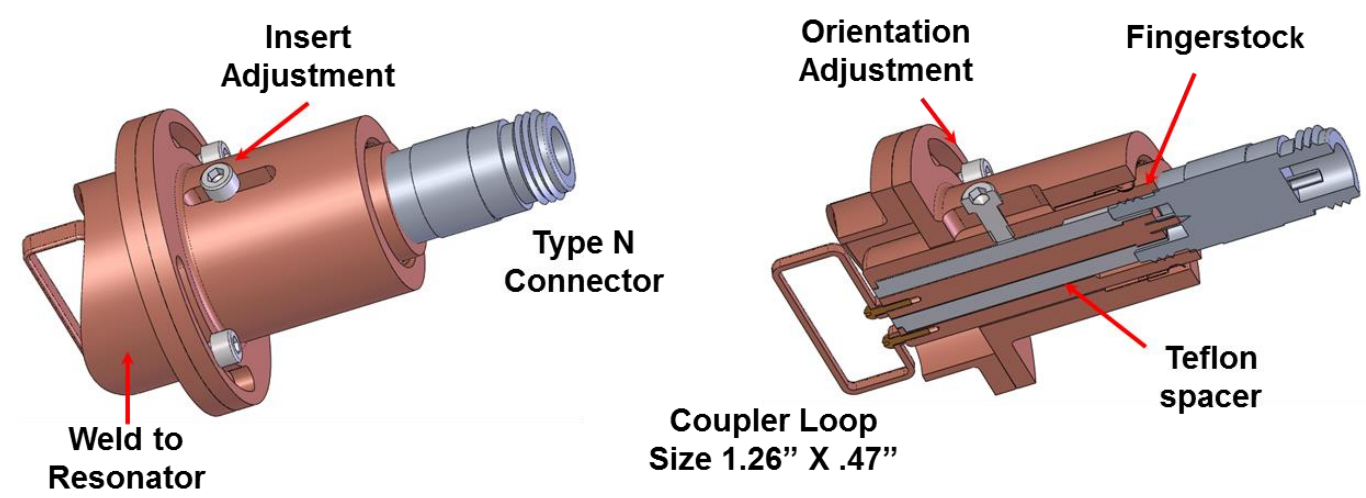

\section{RF probe design}

RF probes have a design similar to that of the power couplers but utilize a smaller loop.
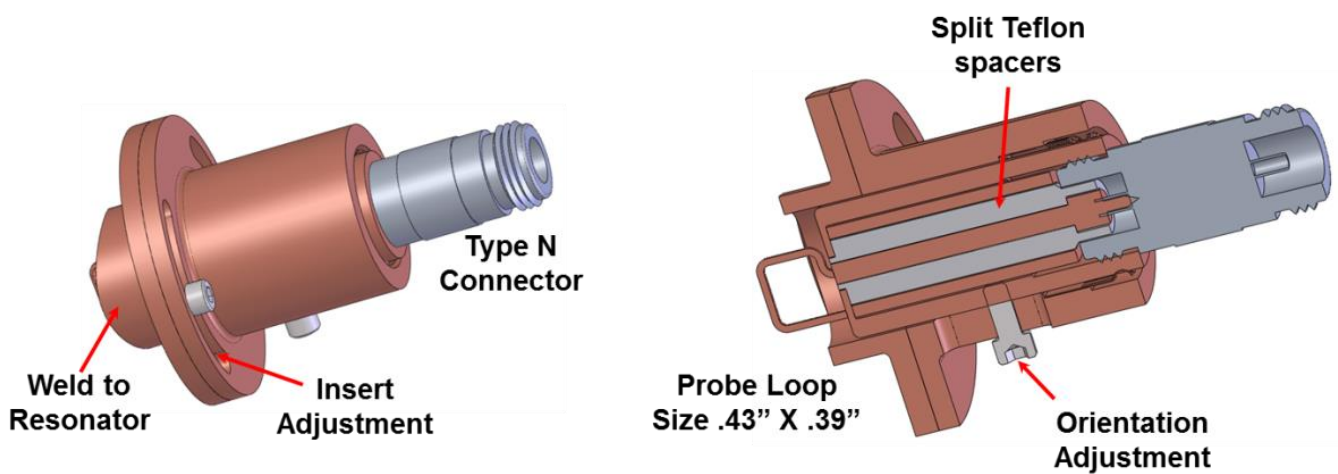

\section{Tuner design}

Three screws were used as tuners. Threaded nipples for the tuners were brazed onto the resonators.

The tuners situated at the top of the resonators (Tuners \#1 and \#2, Figure 1) were not actively used into tuning as their effect is equivalent to changing the length of the resonators. The Tuner \#3 is used to set up and tune the frequency of the $120.75 \mathrm{MHz}$ mode.

\section{RF Modeling}

RF parameters such as the frequency of the modes and their quality factor were simulated using the program SUPERFISH. Each resonator was simulated separately. Figure 6 and Figure 7 show the SUPERFISH models for the short (80.5 MHz) and long (40.25 MHz and $120.75 \mathrm{MHz}$ ) resonators. Table 1 shows simulated RF parameters of the bunchers. To include the coupling between the conical drift tubes, the realistic geometry of the electrodes was modified. The electrodes were oriented along the axis of a simulated resonator instead of the beam axis. The electrode of the other resonator was connected to the vacuum box. Although this model does not fully support the actual geometry of the 
full system, it should reasonably accurately include the effect of the capacitive coupling between resonators. The rexolite spacers were represented by axisymmetric cylinders. The area corresponding to the spokes and the slots provided for the key tool were represented by axisymmetric cylinders with the length of the cylinders scaled down to account for a reduced amount of material due to azimuthal gaps between the spokes. The effect of coupler loops and tuners on the mode frequency was not included in the model. However, the relative effectiveness of tuners and their impact on the modes were studied separately. The transit time factor was simulated using electric fields of the conical electrodes generated by the code POISSON.

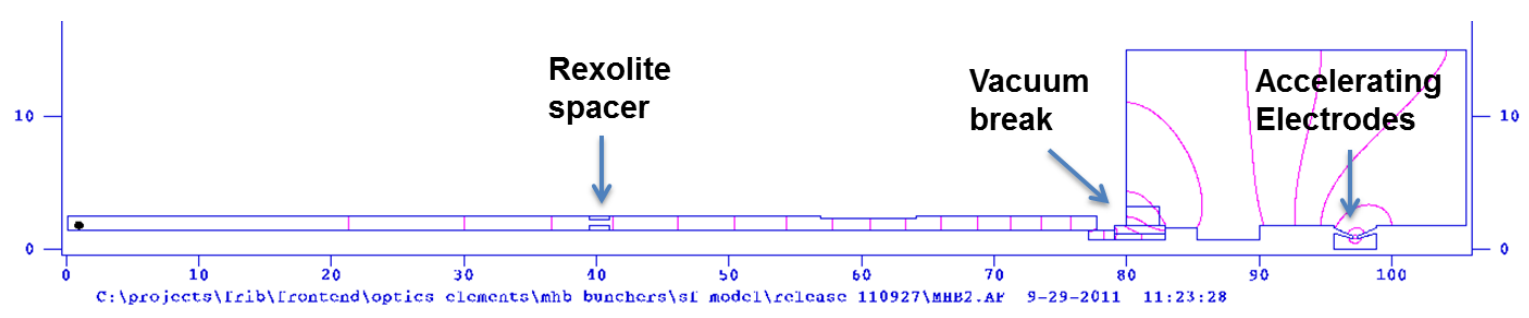

Figure 6: SUPERFISH model of the short $(80.5 \mathrm{MHz})$ resonator.

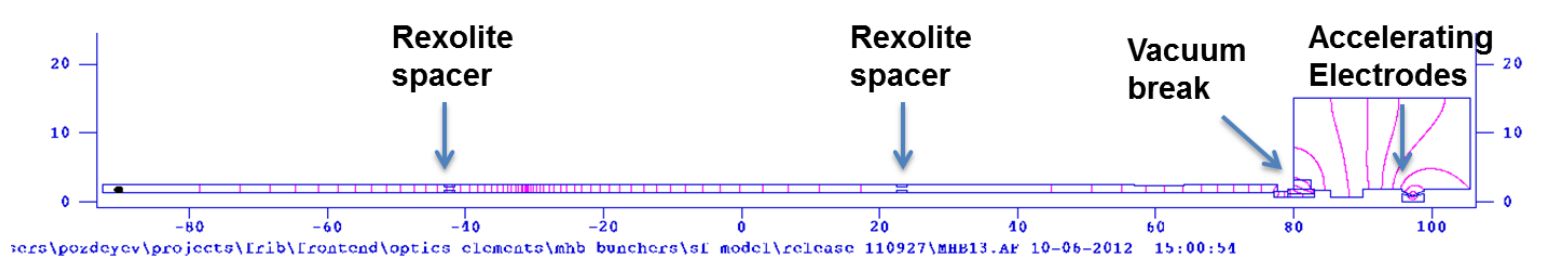

Figure 7: SUPERFISH model of the long (40.25 and $120.75 \mathrm{MHz})$ resonator.

Table 1: Simulated parameters of the MHB. Numbers are given for $40.25,80.5$, and $120.75 \mathrm{MHz}$ respectively.

\begin{tabular}{|l|c|c|c|}
\hline Parameter & \multicolumn{3}{|c|}{ Value } \\
\hline Mode frequency (MHz) & 40.25 & 80.5 & 120.75 \\
\hline Mode quality factor & 882 & 1107 & 1428 \\
\hline Transit time factor (based on Poisson fields) & 0.82 & 0.48 & 0.23 \\
\hline Nominal accelerating mode voltage (including TTF, Volt, U beam) & 1271 & 456 & 150 \\
\hline Voltage on the electrode (V) & 1550 & 950 & 650 \\
\hline Mode power for nominal voltage (W) & 33.3 & 8.7 & 7.8 \\
\hline
\end{tabular}

\section{Tuning strategy}

The buncher design allows for practically independent tuning of all the three modes. The frequency of the $40.25 \mathrm{MHz}$ and $80.5 \mathrm{MHz}$ modes are set by adjusting the length of the resonators. Because the capacitive coupling between the resonators is small no farther adjustment of the smaller resonator (80.5 $\mathrm{MHz}$ ) is required. The frequency of the $120.75 \mathrm{MHz}$ mode can be fine-tuned using tuner 3 (Figure 1) in a range of $300 \mathrm{kHz}$, assuming that the distance between the tuner tip and the central electrode changes from $4 \mathrm{~mm}$ to $20 \mathrm{~mm}$ (completely withdrawn). In principle, the tuning range can be increased if the tuner is brought closer to the central electrode. Coarse tuning of the 120.75 mode frequency can be achieved by changing the length of the upper Rexolite spacer sitting next to the tuner 3 . The sensitivity of the mode frequency to the spacer length is approximately $75 \mathrm{kHz} / \mathrm{mm}$. The impact of tuning the 
120.75 mode on the 40.25 mode using the tuner and the spacer length is small and easily compensated by small adjustments of the length of the long resonator. There is no noticeable impact of tuning 120.75 $\mathrm{MHz}$ mode on the $80.5 \mathrm{MHz}$ mode in the small resonator.

\section{RF tests}

\section{Preliminary measurements and modifications of the bunhcer design}

The bunhcer was assembled and tuned using a NWA. The initial tuning test was conducted with all the Rexolite spacers identical, using the short design as shown in Figure 3. Below are results of the preliminary measurements:

1. The frequency of the $80.5 \mathrm{MHz}$ modes was tuned

2. The long resonator would have to be lowered to the lowest possible position to get the 40.25 $\mathrm{MHz}$ mode close to $40.25 \mathrm{MHz}$. In this position, the $40.25 \mathrm{MHz}$ could be tuned within the bandwidth but not centered.

3. The frequency of the $120.75 \mathrm{MHz}$ mode was too high. The splitting between the modes, $F_{120}-3 F_{40}$, was of the order of $1 \mathrm{MHz}(\sim 1 \%)$. To tune out this difference tuner 3 has to be inserted deeply in the resonator, almost touching the central conductor, which was deemed unfavorable.

4. Coupler $3(120.75 \mathrm{MHz})$ has significant impact on the $40.25 \mathrm{MHz}$ frequency caused by capacitive coupling between the coupler loop and the central electrode.

To address issues 2 and 3 following modifications were made:

1. The length of the long resonator tube was reduced by 1 ", providing additional tuning range. Figure 2 shows the final modified resonator length in the middle of the tuning range.

2. The design of the upper Rexolite spacer was modified to increase the length of the spacer as shown in Figure 3, right. (Initial RF tuning test was made with all small Rexolite spacers shown in Figure 3, left.) The length of the extended solid portion of the tuner was set to $0.494 "$. The length of the spacers was selected assuming the gap between the tip of tuner 3 and the central electrode is $7 \mathrm{~mm}$.

\section{Results of low power measurements and calibration}

The voltage v.s. power curve was calibrated by a direct measurement of the voltage on the electrodes vs RF power. Appendix A shows the setup used for these measurements. The power was provided by a network analyzer, while the voltage on electrodes was measured by a high-impedance RF voltmeter. The power-voltage relation was checked at two levels of power, $0 \mathrm{dBm}$ and $6 \mathrm{dBm}$, to verify consistency of voltage measurements. The voltmeter probe shifted frequencies of the modes. However, this shift did not exceed $5 \%$ and was rather insignificant. The impact of the probe on the mode voltage distribution profile was neglected. The response of the RF pickups was measured and adjusted to give $-40 \mathrm{dBm}$ power at $1 \mathrm{dBm}$ forward power. Table 2 summarizes results of these measurements. 
Table 2: Results of calibration of electrode voltage and RF pickup signal vs RF power.

\begin{tabular}{|l|c|c|c|c|c|c|}
\hline Mode \# & $\begin{array}{c}\text { Nominal } \\
\text { Frequency }\end{array}$ & $\begin{array}{c}\text { Frequency } \\
\text { with probe }\end{array}$ & $\begin{array}{c}\mathbf{Q} \\
\text { Measured }\end{array}$ & $\begin{array}{c}\mathbf{P}_{\text {NWA }} \\
(\mathbf{d B m})\end{array}$ & $\begin{array}{c}\mathbf{V}_{\text {electrode,peak }}(\mathbf{V}) \\
\text { measured }\end{array}$ & $\begin{array}{c}\mathbf{P}_{\text {pickup, }} \\
(\mathbf{d B m})\end{array}$ \\
\hline 1 & 40.25 & 39.16 & 515 & 0 & 7.56 & -40.2 \\
\hline 2 & 80.5 & 76.3 & 325 & 0 & 7 & -40.2 \\
\hline 3 & 120.75 & 115.1 & 244 & 0 & 5.46 & -40.2 \\
\hline
\end{tabular}

The required power can be estimated by direct scaling of the measured voltage and power from Table 2 . Also, the required power can be estimated using results of simulations shown in Table 1 and the measured quality factor from Table 2, assuming that SUPERFISH can accurately simulate the stored energy but not the quality factor because of design details such as RF contacts. Table 3 shows the nominal mode power for uranium beam estimated by direct scaling of the measured P-V curve from Table 2 (P1). The table also shows the estimated nominal power obtained by scaling the simulated power from Table 1 using the measured quality factor from Table 2 (P2). The difference between two different estimates reaches a factor of 3 . The source of this discrepancy is not fully understood and has to be addressed by more measurements. However, it is necessary to note that the maximum estimated value is still factor of two lower than available RF power (also see the description of the high power test).

Table 3: Nominal mode power for uranium beam estimated by direct scaling of the measured $P-V$ curve from Table $2\left(P_{1}\right)$ and by scaling the simulated power from Table 1 using the measured quality factor from Table $2\left(\mathrm{P}_{2}\right)$.

\begin{tabular}{|l|c|c|}
\hline Mode \# & $\mathrm{P}_{1}$ (nominal, $\mathrm{W}$ ) & $\mathrm{P}_{2}$ (nominal, $\mathrm{W}$ ) \\
\hline 1 & 42 & 56 \\
\hline 2 & 18 & 29 \\
\hline 3 & 14 & 45 \\
\hline
\end{tabular}

The buncher was subjected to mechanical perturbations typical for tuning and handling activities (pushed and shaken). The corresponding frequency changes were negligible comparatively to the bandwidth of the buncher.

\section{High power test}

The high power test was conducted to attest the operational stability of the buncher at a high level of RF power. Appendix A shows the setup of the test, which is similar to the final operational configuration.

First, the buncher was tested "cold". The RF power was turned on for a short period of time after the buncher was tuned at a low level. The combined power level for all modes during the test was 180$190 \mathrm{~W}$, approximately a factor of 2 exceeding the expected operational power level. During the cold test the buncher was practically at the ambient temperature. The forward and reflected power levels were measured. After letting the buncher run at a high power level for an extended period of time (up to 70 hours), the forward and reflected power were measured again (the "hot" test). Figure 8 shows the equilibrium temperature distribution under the "hot" conditions. Table 4 shows the forward and reflected power under different conditions. Small changes in the reflected power under "cold" and "hot" conditions demonstrate high stability of the mode frequency under power load. Direct measurements of 
the frequency demonstrated negligible variations in the frequency of $40.25 \mathrm{MHz}$ and $80.5 \mathrm{MHz}$ modes. The frequency of the $120.75 \mathrm{MHz}$ mode shifted by approximately $80 \mathrm{kHz}(1 \mathrm{e}-3)$, which is smaller than bandwidth of the mode and should not present a problem for operations. The observed detuning is consistent with changes of the Rexolite permittivity with temperature. The design of the buncher can be adapted to allow for blowing a small amount of cooling air through the resonators, if necessary.

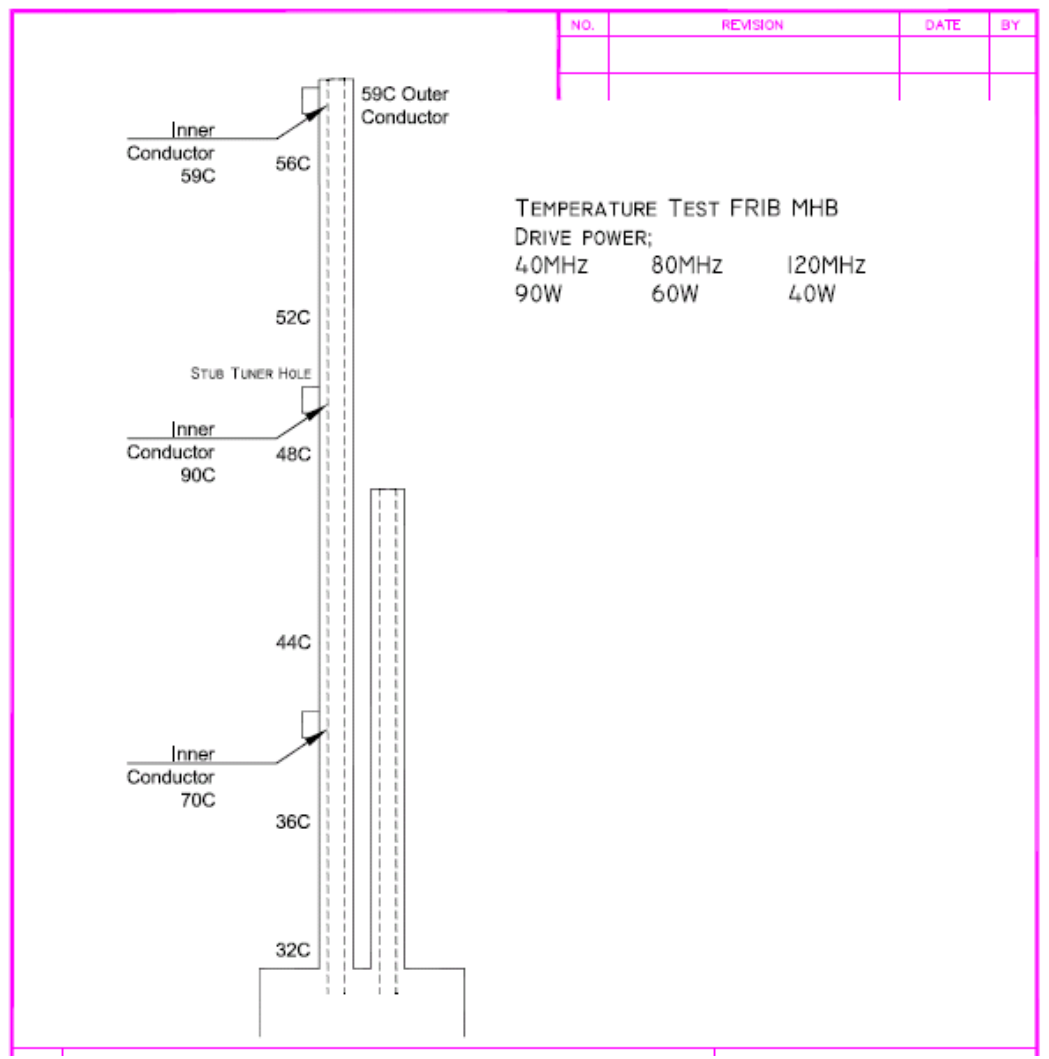

Figure 8: Equilibrium temperature distribution of the buncher running at the total power of $190 \mathrm{~W}$, approximately factor of 2 higher than the required nominal power. Under this high power, the temperature stays within the operational region of Rexolite and is not expected to strongly affect the frequency of the modes.

Table 4: Forward and reflected power (in W) under different conditions. Small changes in reflected power under "cold" and "hot" conditions demonstrate stability of the mode frequency.

\begin{tabular}{|c|c|c|c|}
\hline Cold & $\mathbf{4 0 . 2 5}$ & $\mathbf{8 0 . 5}$ & $\mathbf{1 2 0 . 7 5}$ \\
\hline Forward & 20 & 20 & 20 \\
\hline Reflected & 3 & 5 & 2 \\
\hline Cold & $\mathbf{4 0 . 2 5}$ & $\mathbf{8 0 . 5}$ & $\mathbf{1 2 0 . 7 5}$ \\
\hline Forward & 80 & 60 & 40 \\
\hline Reflected & 10 & 16 & 4 \\
\hline Cold & $\mathbf{4 0 . 2 5}$ & $\mathbf{8 0 . 5}$ & $\mathbf{1 2 0 . 7 5}$ \\
\hline Forward & 100 & 40 & 60 \\
\hline Reflected & 10 & 13 & 4 \\
\hline Hot & $\mathbf{4 0 . 2 5}$ & $\mathbf{8 0 . 5}$ & $\mathbf{1 2 0 . 7 5}$ \\
\hline Forward & $>100$ & 44 & 58 \\
\hline Reflected & 8 & 13 & 4 \\
\hline
\end{tabular}




\section{Summary and future work}

The buncher was designed, manufactured, assembled, and tested. Tuning of the buncher showed that the two resonators are well decoupled making tuning of the modes easier. Tests showed that the buncher is able to reach the desirable electrode voltage with available RF power and is stable under a high RF power load. Also, the buncher was stable under mechanical disturbances that are likely to happen during buncher handling and tuning. Based on the test results, we can conclude with a high degree of confidence that the buncher will satisfy operational requirements and successfully operate in the FRIB beam line.

There are several questions left, addressing which prior to the installation of the buncher in the tunnel is either required or would be beneficial:

1. The low power test indicated a discrepancy between the calibrated power-voltage curve and the measured value of the quality factor. Although sufficient RF power is available regardless which method is used to calculate the required RF power, additional measurements are strongly desirable to resolve this discrepancy.

2. It is desirable to test the buncher with a beam to attest voltage calibration and check stability of the buncher parameters against possible beam losses. We are looking for an opportunity to test the multi-harmonic buncher with beam using either the ReA3 or ARTEMIS B off-line facility, which is currently under construction.

3. The alignment of the electrodes relatively to the outside alignment fixtures must be measured and documented prior to storing the buncher.

\section{References}

1. Q. Zhao+, V. Andreev, J. Brandon, G. Machicoane, F. Marti, J. Oliva, J. Ottarson, J. Vincent, "DESIGN AND TEST OF THE TRIPLE-HARMONIC BUNCHER FOR THE NSCL REACCELERATOR", Proceedings of LINAC08, Victoria, BC, Canada, p.948

\section{Appendix A}

Low power test setup, test procedure, and equipment

Equipment used;

1) VNA, (Dan's Agilent $8753 \mathrm{ES}$ in this case)

2) Boonton RF millivolt RF meter with 100:1 high impedance adapter.

3) Tomco TE1000 RF Impedance Analyser with the 2.2 .7 software. (Not really necessary).

4) EMPower 20 to $125 \mathrm{MHz} 100 \mathrm{~W}$ RF amplifier.

Procedure;

1. Set the VNA up on one of the three frequencies.

2. Measure S11 and set the marker search for min.

3. Sweep about a $5 \mathrm{MHz}$ band and center the fundamental frequency.

4. Adjust the coupling for lowest return.

(a) $80.5 \mathrm{Mhz}$ coupling loop is inline with the inner conductor and almost touching. 
i. Apply some Kapton tape to the surface of all the coupling loops so it won't short on the inner conductor.

(b) $120.75 \mathrm{MHz}$ loop is inline with the inner conductor and almost touching.

(c) $40.25 \mathrm{MHz}$ is

5. Switch (or dual screen) to measure S21.

(a) Set drive power to $0 \mathrm{dBm}$.

(b) Set sweep to manual and 10 or 20 second sweep.

(c) Marker search max.

6. Probe the electrode with the Boonton as close to the gap as possible.

7. Determine the VRMS at resonance as the sweep should be slow enough to see the peak on the meter.

8. Record the VRMS and convert to $V$ peak.

9. Set the drive power to $+6 \mathrm{dBm}$ and see that the VRMS will double. (Four times the power to double the voltage).

10. Go back to $0 \mathrm{dBm}$ (can be any drive power actually in S21) drive and set the coupling on the pick up to exactly $-20 \mathrm{dBm}$.

11. Add a type $N 20 \mathrm{~dB}$ attenuator so the coupling factor from drive power to pickup is $-40 \mathrm{~dB}$. 

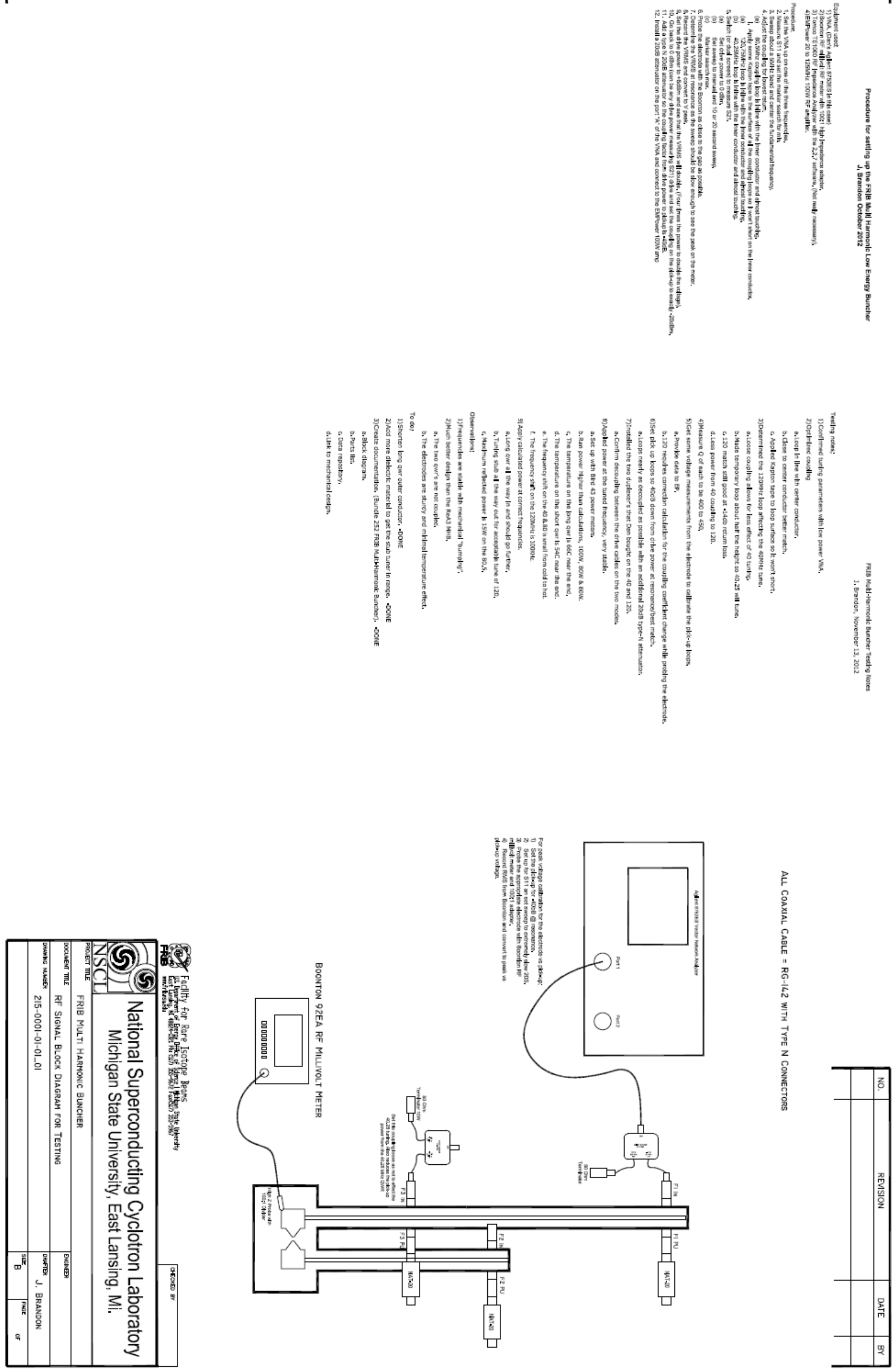
High power test setup

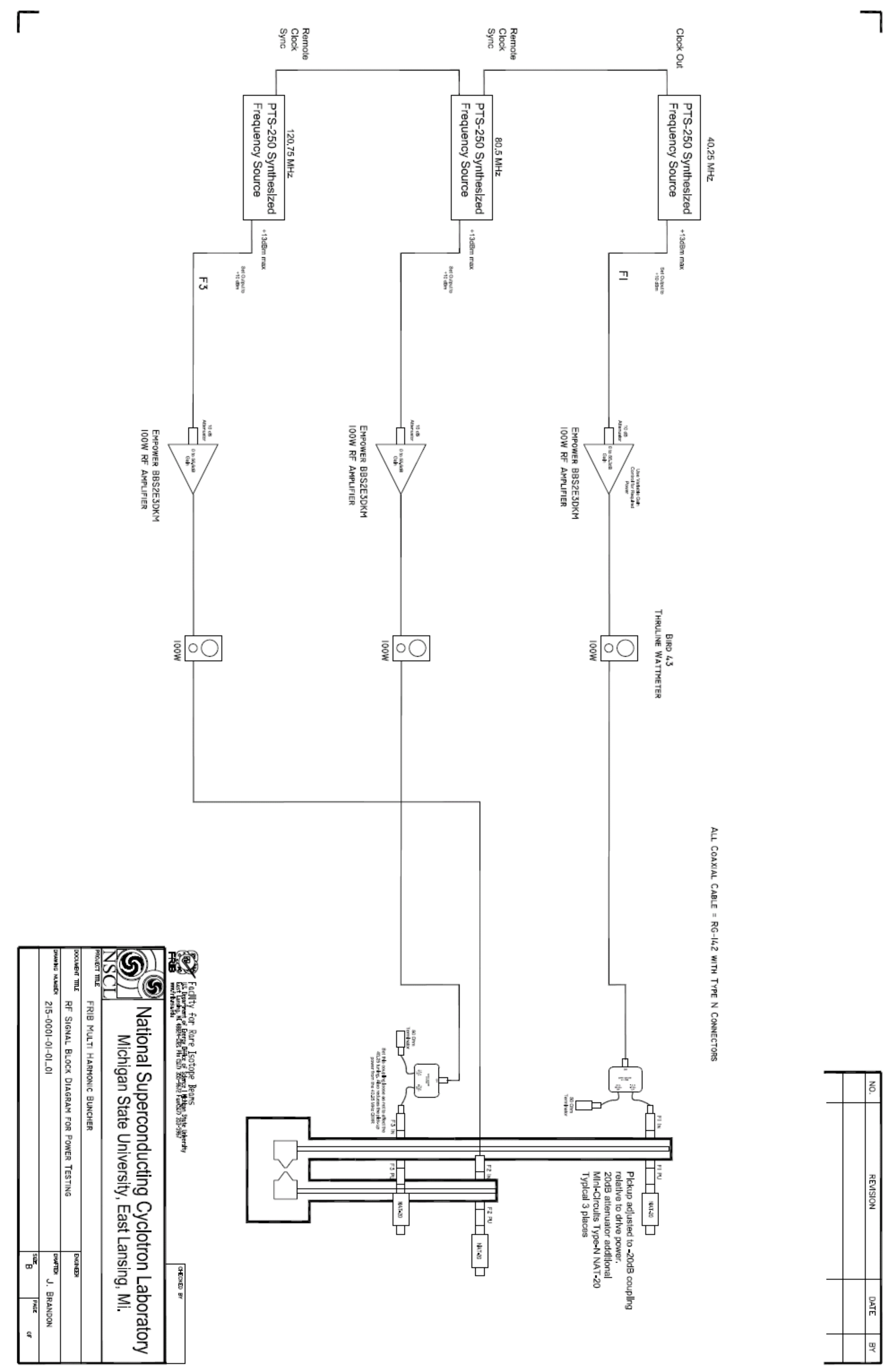

